\title{
Digital Signal Processing Approach in Air Coupled Ultrasound Time Domain Beamforming
}

\author{
Krzysztof HERMAN $^{(1)}$, Tadeusz GUDRA ${ }^{(1)}$, Joanna FURMANKIEWICZ ${ }^{(2)}$ \\ (1) Faculty of Electronics, Wroclaw University of Technology \\ Wybrzeze Wyspianskiego 27, 50-320 Wroclaw, Poland; e-mail: Krzysztof.Herman@pwr.wroc.pl \\ ${ }^{(2)}$ Faculty of Behavioural Ecology, University of Wroclaw \\ Pl. Uniwersytecki 1, 50-137 Wroclaw, Poland
}

(received October 12, 2012; accepted December 12, 2013)

\begin{abstract}
The work presents the results of experimental study on the possibilities of determining the source of an ultrasonic signal in two-dimensional space (distance, horizontal angle). During the research the team used a self-constructed linear array of MEMS microphones. Knowledge in the field of sonar systems was utilized to analyse and design a location system based on a microphone array. Using the above mentioned transducers and broadband ultrasound sources allows a quantitative comparison of estimation of the location of an ultrasonic wave source with the use of broadband modulated signals (modelled on bats' echolocation signals) to be performed. During the laboratory research the team used various signal processing algorithms, which made it possible to select an optimal processing strategy, where the sending signal is known.
\end{abstract}

Keywords: beamforming, air-coupled ultrasounds, DSP, bat signals, direction of arrival.

\section{Introduction}

Echolocation inspired devices, that function as a sonar in a gas medium have been in development for many years. Due to problems related to generating, transmiting and detecting ultrasonic signals in air (difference between acoustic impedance of the source and gas medium, high attenuation coefficient in gases (BASS et al., 1995; EvANs et al., 1972) these are usually designs with one or two sensors that generate a constant frequency ultrasonic wave. Examples of such devices were described in works (KAY, 1964) and (Borenstein, Koren, 1988). The appliances can be widely used in robotics. They use simple ultrasonic sensors assisted by more or less advanced scanning pulse generation installations and echo signal processing systems. Advanced signal modelling at detection was presented in work (ShI, HorIUCHI, 2007). The described signal processing method is based on bats' neuronal system.

Advanced signal processing with extraction of the parameters of signal amplitude spectrum and an attempt to correlate them with the direction of incidence of ultrasonic wave was described in studies
(Peremans et al., 1993; Schillebeeckx, 2008). The works cover the problem of imaging three-dimensional space using receiving transducers located in artificial auricles modelled on bats' auricles. The use of multichannel techniques and dynamic beamforming (Krim,Viberg, 1996; Van Veen, Buckley, 1988) in ultrasonic devices operating in air environment is covered in works (WebB, Wykes, 1996; Wykes et al., 1993; Tsung, Wykes, 1997; Strakowski et al., 2006; Salamon et al., 1997; Medina, Wykes, 2001; HARPut, Bozkut, 2008). The systems described in those studies are based on signals with the constant frequency of $18.5-100[\mathrm{kHz}]$. This is primarily due to a limitation related to transducer design. Introduction of transducers made of new materials like EMFi (ElectroMechanical Film, described in study (PAAJANEN et al., 2000); application of the material in a transducer intended for operation in air environment is covered in (EALO et al., 2008)) or MEMS makes it possible to miniaturize the transducers, but also to use modulated frequency signals. For a more in-depth analysis of echolocation systems based on bats' signals see work (GUDRA et al., 2011). It describes the results of experiments, which present the properties of the 
developed microphone array for various measurement signals and signal processing algorithms. The experiments involved estimation of the location of a source of a known ultrasonic signal in horizontal plane and estimation of source-matrix distance. Authors used bats-like signals in the experiments in order to examine matrix localization performance. Signal processing used during the research was simply delay-and-sum beamforming with modifications (described in detail in Sec. 4).

\section{Air operating ultrasound microphone array based on MEMS sensors}

The main element of the system locating the source of ultrasonic waves is a planar array with 64 MEMS microphones (Micro Electro-Mechanical Systems). A view of one half of the array is shown in
Fig. 1. Two microphone antennae consisting of $32 \mathrm{mi}-$ crophones each were located on the even surface of the array. In all experiments one line of 32 sensors was used for testing.

For a microphone antenna with this kind of geometry the directivity function is given by dependence:

$$
J(\alpha, M)=\frac{\sin \left(M \cdot \pi \cdot \frac{D}{\lambda} \cdot\left(\sin (\alpha)-\sin \left(\alpha_{k}\right)\right)\right)}{M \cdot \pi \cdot \frac{D}{\lambda} \cdot\left(\sin (\alpha)-\sin \left(\alpha_{k}\right)\right)},
$$

where $M$ stands for number of sensors in linear array, $D$ is inter sensor spacing, $\alpha$ is a horizontal angle and $\alpha_{k}$ is a steering angle.

Figure 2 shows a graph of the directivity function of the antenna. A equation on the Fig. 2 also shows a crucial characteristic: increase of the width of the main lobe with increasing beam steering angle.

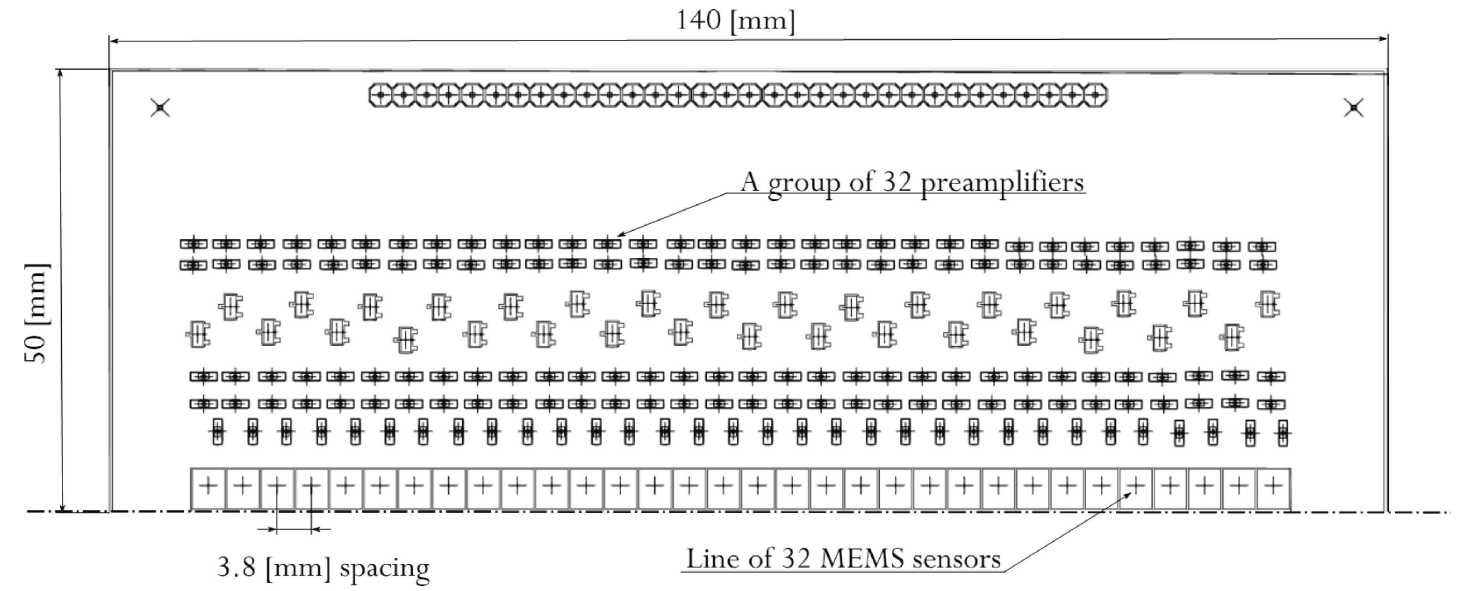

Fig. 1. Vertical projection of the microphone array. The array is symmetric along horizontal axis.

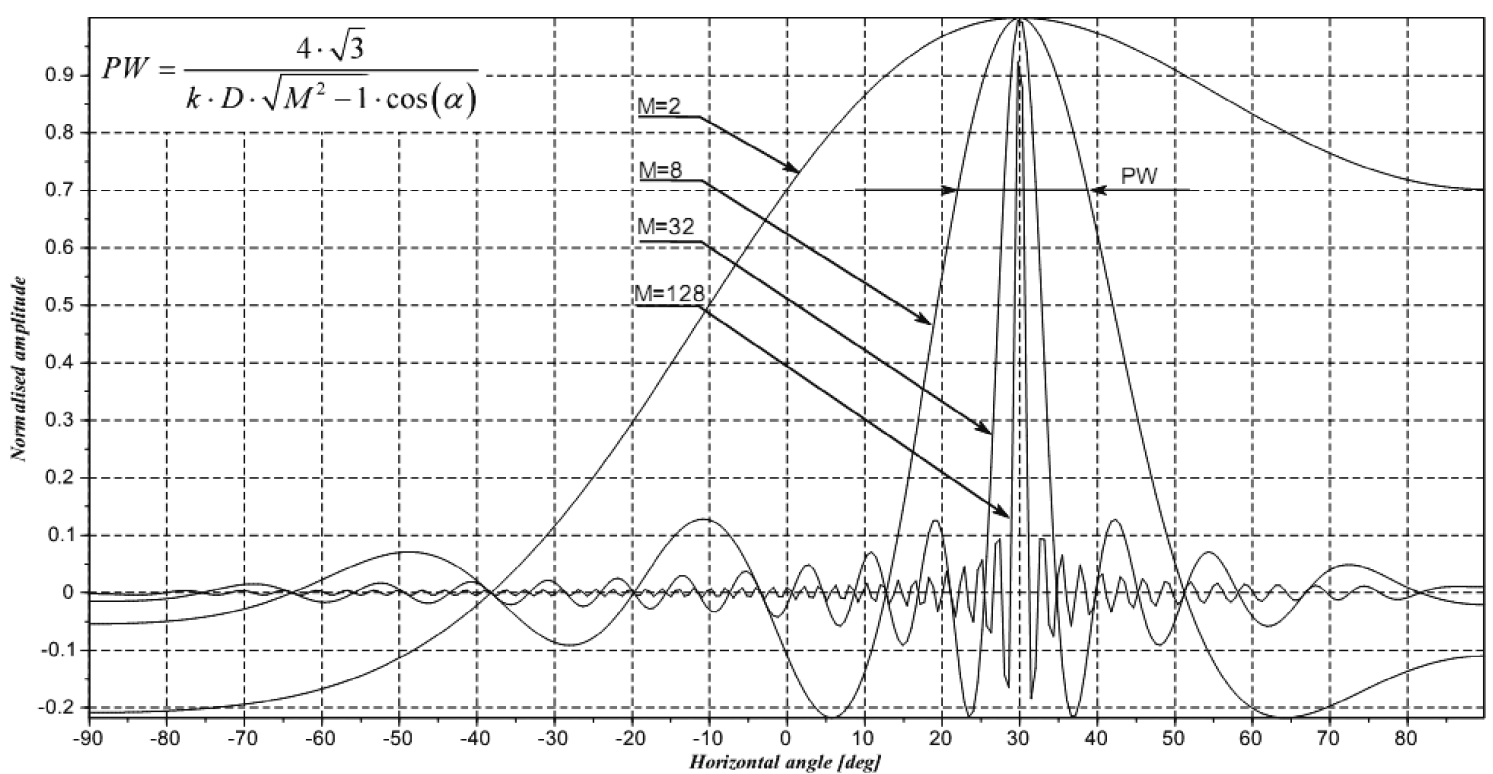

Fig. 2. Directivity function of the array for beam steering equal to 30 [deg]. Also marked is the main lobe's width as a parabolic approximation (Johnson, Dudgeon, 1993). PW-Parabolic Width, $k$-wavenumber. 
The geometry of MEMS sensors, the array is made of, dictate maximum limit frequency for the antenna's operation. For $D=3.8[\mathrm{~mm}]$ the equation gives $44.736[\mathrm{kHz}]$

$$
\lambda \geq 2 \cdot D
$$

Since the linear array has 32 sensors, the team opted for sequential measurement signal reading using a system shown in Fig. 3. Sequential reading sensors signals is based on assumption that the position of measured object is stable. A multiplexing circuit was applied to measure 32 signals using 4 input analog to digital converter. Since authors use 8 multiplexers a demultiplexer circuits was applied to simplify address generation by generating only 6 bits (three for multiplexer selection and three for a particular input selection).

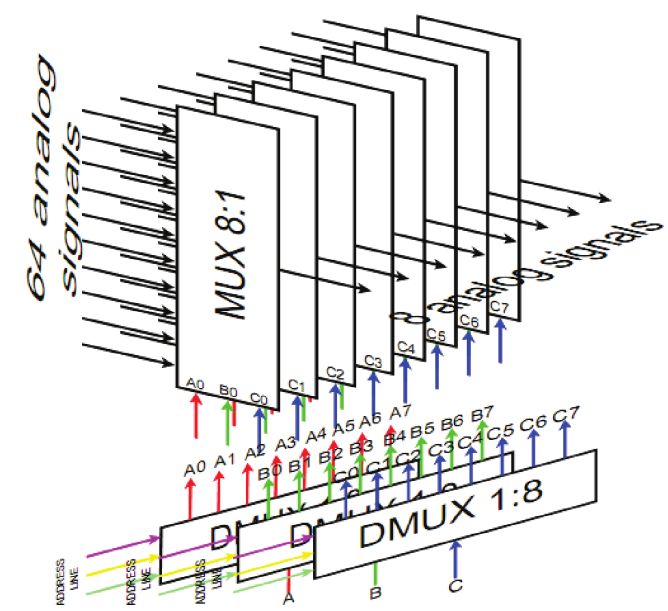

Fig. 3. Schematic of signal muxing from MEMS sensors.
The whole muxing process is managed by a measurement controlling microprocessor, which is also responsible for triggering the channel switching sync signal. In our study only half of sensors were used - one 32 element linear array.

\section{Source localization measurements setup}

Estimation of spatial resolution and the distance between the source and the array was performed using the arrangement shown in Fig. 4.

For a given location of ultrasonic wave source the team performed 5 measurements of the signal from each microphone to improve signal to noise ratio. The pulse sent by a computer controlled NI-DAQ 6259M measurement card is properly described by the equation Eq. (3) for constant sine wave CF and Eq. (4) for frequency modulated wave FM.

$$
\begin{aligned}
& x_{t C F}(n)=A \cdot \sin \left(2 \cdot \pi \cdot \frac{f}{f_{s t}} \cdot n\right), \\
& x_{t F M}(n)=A \cdot \sin \left(2 \cdot \pi \cdot\left(\frac{f}{f_{s t}} \cdot n-\frac{\gamma}{f_{s t}} n^{2}\right)\right) .
\end{aligned}
$$

The parameters of synthesis of signals are: sampling frequency $f_{s t}=1[\mathrm{MHz}]$, signal base $(\mathrm{CF})$ or maximum (FM) frequency $f$ equal to $40[\mathrm{kHz}]$ and frequency modulation index $\gamma$ equal to $10[\mathrm{~Hz}]$. In case of CF signal, the number of samples of the synthesised signal is 1000, and in case of FM signal that number is 2000 .

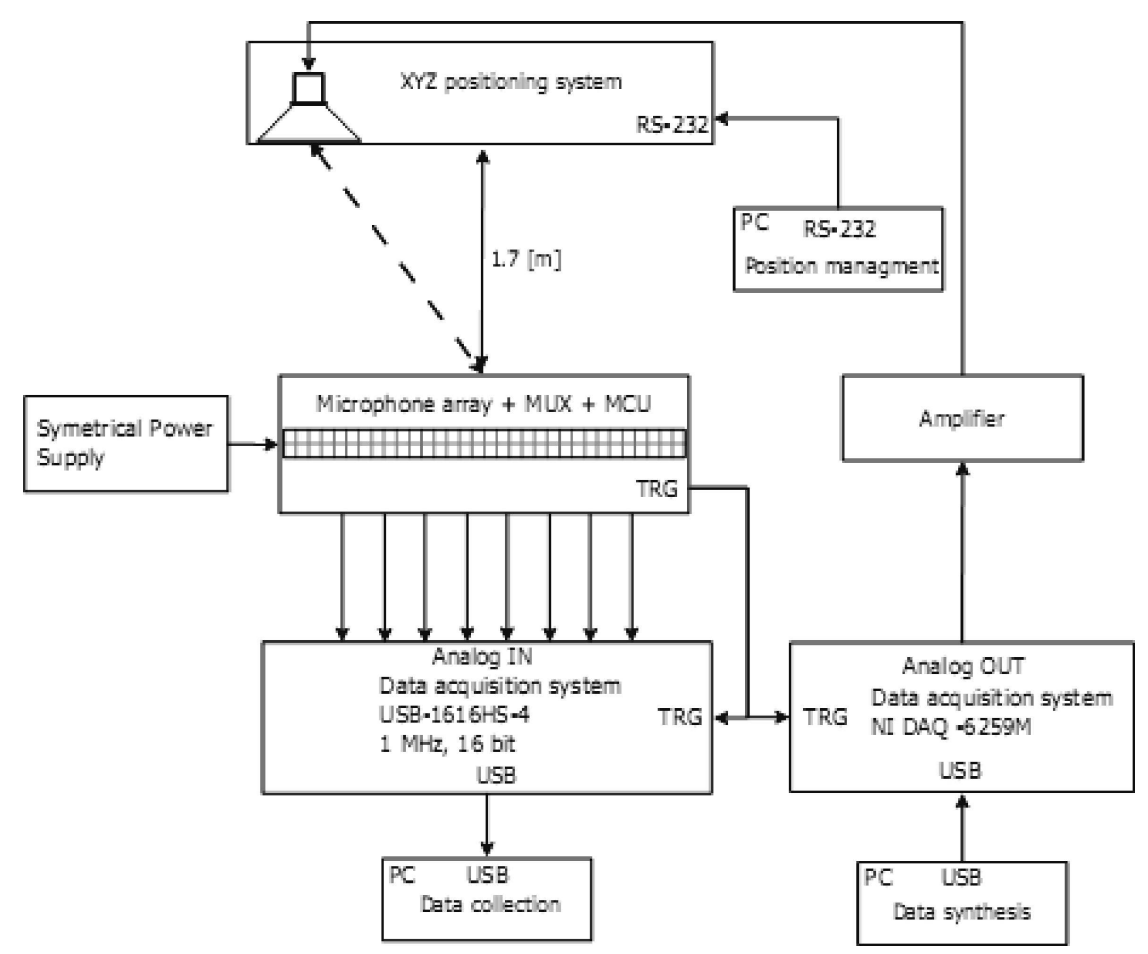

Fig. 4. Diagram of the measurement setup. 


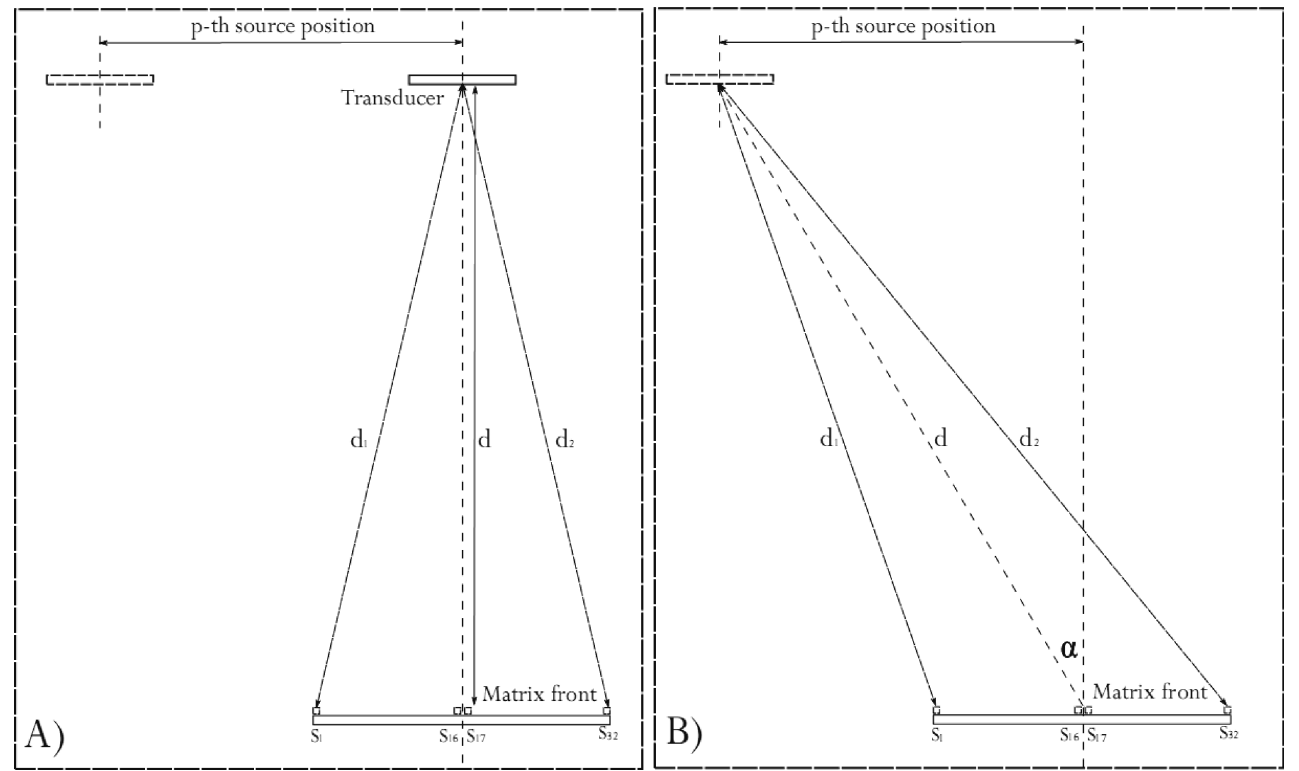

Fig. 5. Schematic of measurement geometry for estimation of ultrasonic wave incidence angle. Schematic A shows the nominal position of the source, at which the incidence angle is $\alpha=0$. Schematic B shows the $p$-th source position; the value of angle $\alpha$ is estimated for this position.

Analogue signals generated by the NI card were passed on to an analogue amplifier, which powered the ultrasonic transducer. The source of CF signals was a Tucker-Davis Technologies ES1 electrostatic transducer powered by a dedicated ED1 amplifier. In case of FM signals the team used a Scan-Speak R2904 dynamic transducer powered by a dedicated amplifier.

The whole system was specifically calibrated to achieve signal $1\left[\mathrm{~V}_{p p}\right]$ on the inputs of the Measure- ment Computing MC-1616HS-4 recording card, in the nominal location of the ultrasonic wave source. The signals were sequentially recorded from 32 microphones at the sampling frequency of $250[\mathrm{kHz}]$ and resolution of $14 \mathrm{bits} / \mathrm{sample}$. In order to facilitate future analysis of the signals, each measurement was recorded as a *.wav file. The geometry of the measurement setup is shown in Fig. 5 (figures are not drawn to scale).

Signals obtained using a TDS1002B oscilloscope are shown in Fig. 6. You can see differences between
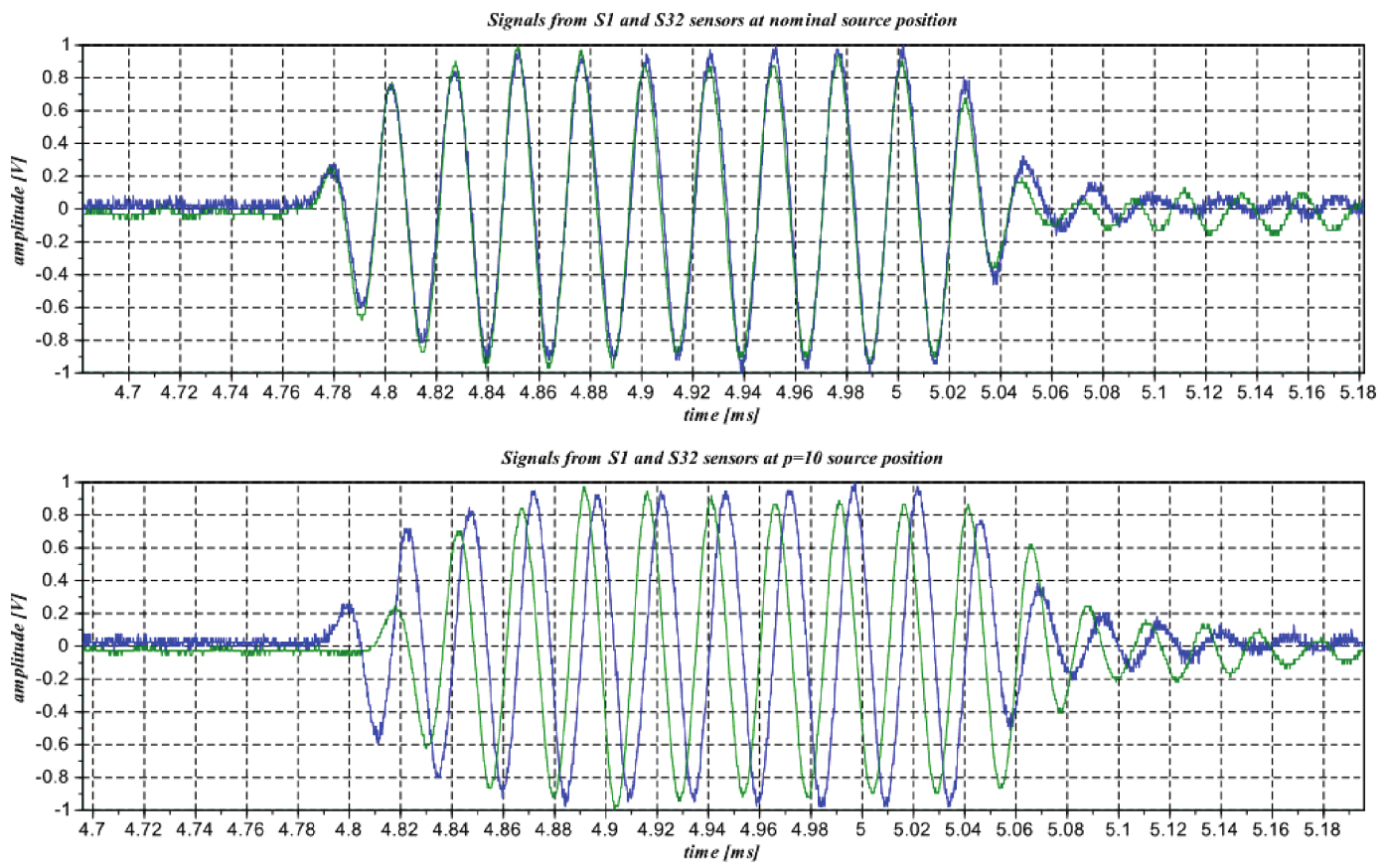

Fig. 6. Signals obtained from the utmost sensors of the $S_{1}$ and $S_{32}$ array for to signal source positions: nominal and horizontally shifted by $10[\mathrm{~cm}]$. 
phases resulting from the shift of the signal source from nominal position to one 10 [cm] away in horizontal plane. Every $p$-th position corresponded to linear shift of $10[\mathrm{~mm}]$, which for a distance of $d=1.7[\mathrm{~m}]$ translates into angular displacement in accordance with the dependence Eq. (5) that equals 0.337 degree.

$$
\alpha(p)=\arctan \left(\frac{p}{100 \cdot d}\right) \cdot \frac{180}{\pi} .
$$

When testing in-depth resolution, the team used a shift with resolution of $0.5[\mathrm{~mm}]$, which in laboratory conditions, at the temperature of about 24 degrees translates to time differences equal to $1.44 \times$ $10^{-6}[\mathrm{~s}]$.

\section{Digital signal processing methods}

The signals obtained during measurements are time series with the length of $N=2000$ samples, obtained during digitisation of the transducer signal with sampling frequency $f_{s}$ of $250[\mathrm{kHz}]$ and resolution of 14 bits/sample. Preprocessing of the obtained results is done by averaging 5 measurements for a given sensor and source position Eq. (6):

$$
\widehat{x}_{m, p}(n)=\frac{1}{5} \sum_{u=0}^{4} x_{m, p, u}(n),
$$

where parameter $m$ indicates successive sensors in $m=1 \ldots 32$ range and parameter $\mathrm{p}$ indicates successive measurement in $p=0$. .45 range, eliminating constant component Eq. (7) from a signal obtained in this manner and filtering low-pass Eq. (8) using a FIR type filter

$$
\begin{aligned}
& \widehat{\widehat{x}}_{m, p}(n)=\widehat{x}_{m, p}(n)-\frac{1}{N} \sum_{n=0}^{N-1} \widehat{x}_{m, p}(n), \\
& x_{m, p}(n)=\sum_{l=0}^{L-1} \widehat{x}_{m, p}(n-l) \cdot a_{l},
\end{aligned}
$$

where $a_{l}$ are the coefficients of the FIR low-pass filter. The low-pass digital FIR filter is used to improve signal to noise ratio and in case of upsampling data operates as an interpolation filter.

After the signals have been readied as described above, they were processed using delay-and-sum beamforming. Due to the directional properties of a flat linear antenna, shown in Fig. 2, it can be assumed that the tested antenna's beam width is 4.5 [deg]. The team tested spatial resolution for the purpose of estimation of horizontal angle for signals sampled at the frequency of $f_{s}$. They also tested spatial resolution for signals interpolated using the FIR filter in accordance with the dependence Eq. (9):

$$
\widehat{f}_{s}=f_{s} \frac{c}{d} \cdot \frac{180}{\pi \cdot \phi}
$$

where $\phi$ is the width of the main lobe of the directional characteristics of the antenna, which in this case is approximated with PW (Parabolic Width) parameter. Delay-and-sum beamforming equation is given by Eq. (10)

$$
y_{k, p}(n)=\sum_{m=0}^{M-1} x_{m, p}\left(n-\frac{m \cdot d}{c} \sin \left(\varphi_{k}\right)\right)
$$

where $k$ indicates successive beam steered by angle $\Delta \varphi_{k}$.

As the next stage of the research beamforming was applied to a signal that was a result of a correlation of the transmitted and received signal, in accordance to Eq. (11)

$$
R_{x_{s}, x}(m)=\frac{1}{N} \sum_{n=1}^{M-m+1} x_{s}(n) \cdot x(n+m+1)
$$

for $m=1,2,3 \ldots, N+1$.

\section{Source localization measurements results}

\subsection{Results obtained for constant frequency signals}

Measurement results obtained for constant frequency signal with delay-and-sum beamforming algorithm are shown in Fig. 7. The graphs present the estimated ultrasonic wave incidence angle in function of actual angular position of the source calculated using Eq. (5). Calculation results are presented for two different values of angle $\Delta \varphi_{k}$ the successive beams were steered by.

As before Fig. 8 shows results of estimation of DOA. The difference is that this time the results were obtained using sample interpolation algorithm in accordance with Eq. (9). As in the case of beamforming with no interpolation, the team performed calculations for two different angle values $\Delta \varphi_{k}$.

Figure 9 shows the results of estimation of ultrasonic wave incidence angle calculated using correlation of the transmitted and received signal and applying beamforming to the obtained time series. Similarly Fig. 10 shows the same result set, the only difference being that, these were obtained using upsampling and interpolation in addition. 

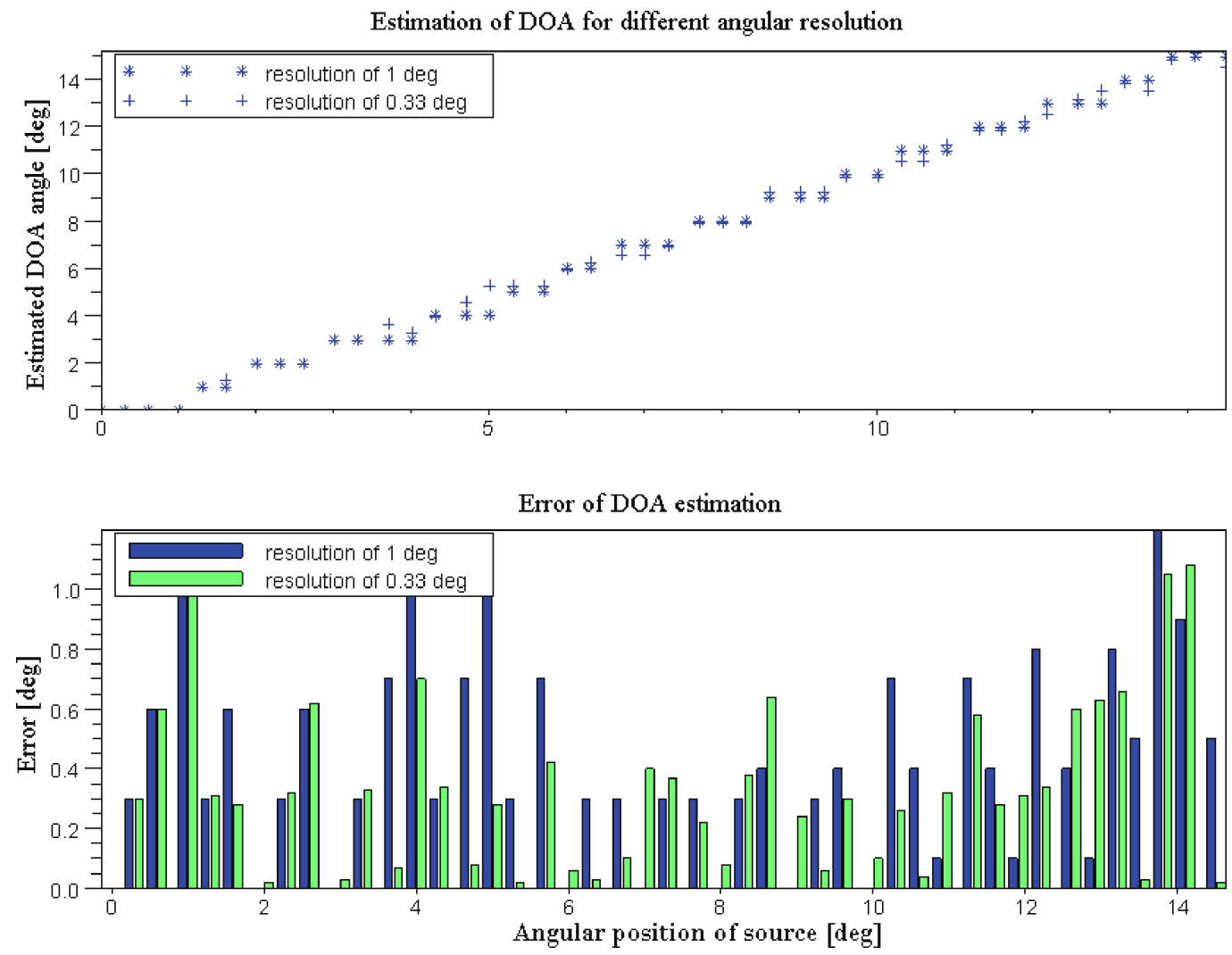

Fig. 7. Estimation results of direction of arrival (DOA) of ultrasound wave incidence for various source positions and various values of beam steering step. Bar charts show estimation error.
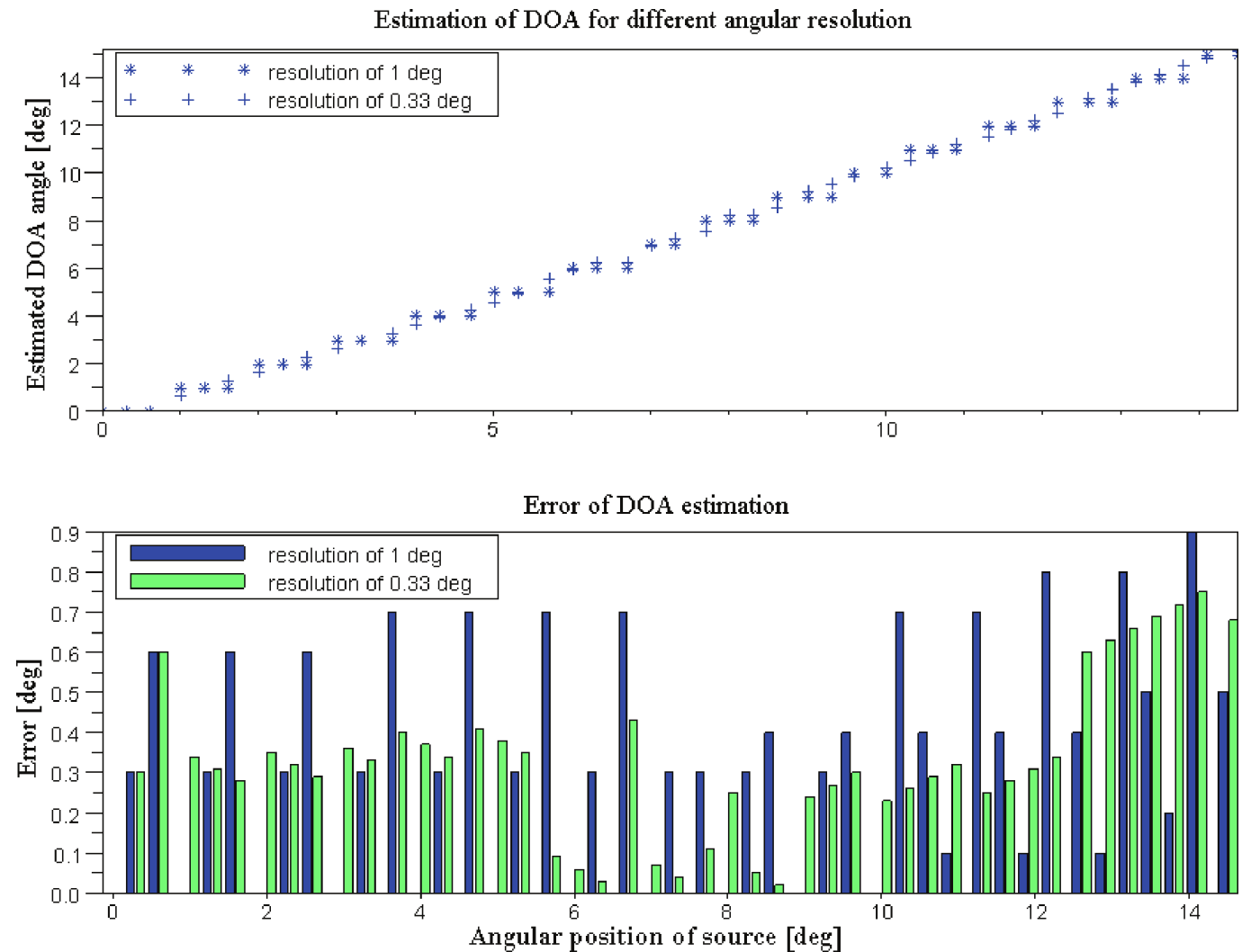

Fig. 8. Estimation results of direction of arrival (DOA) of ultrasound wave for various source positions and various values of beam steering step, when interpolated beamforming was used. Bar charts show estimation error. 

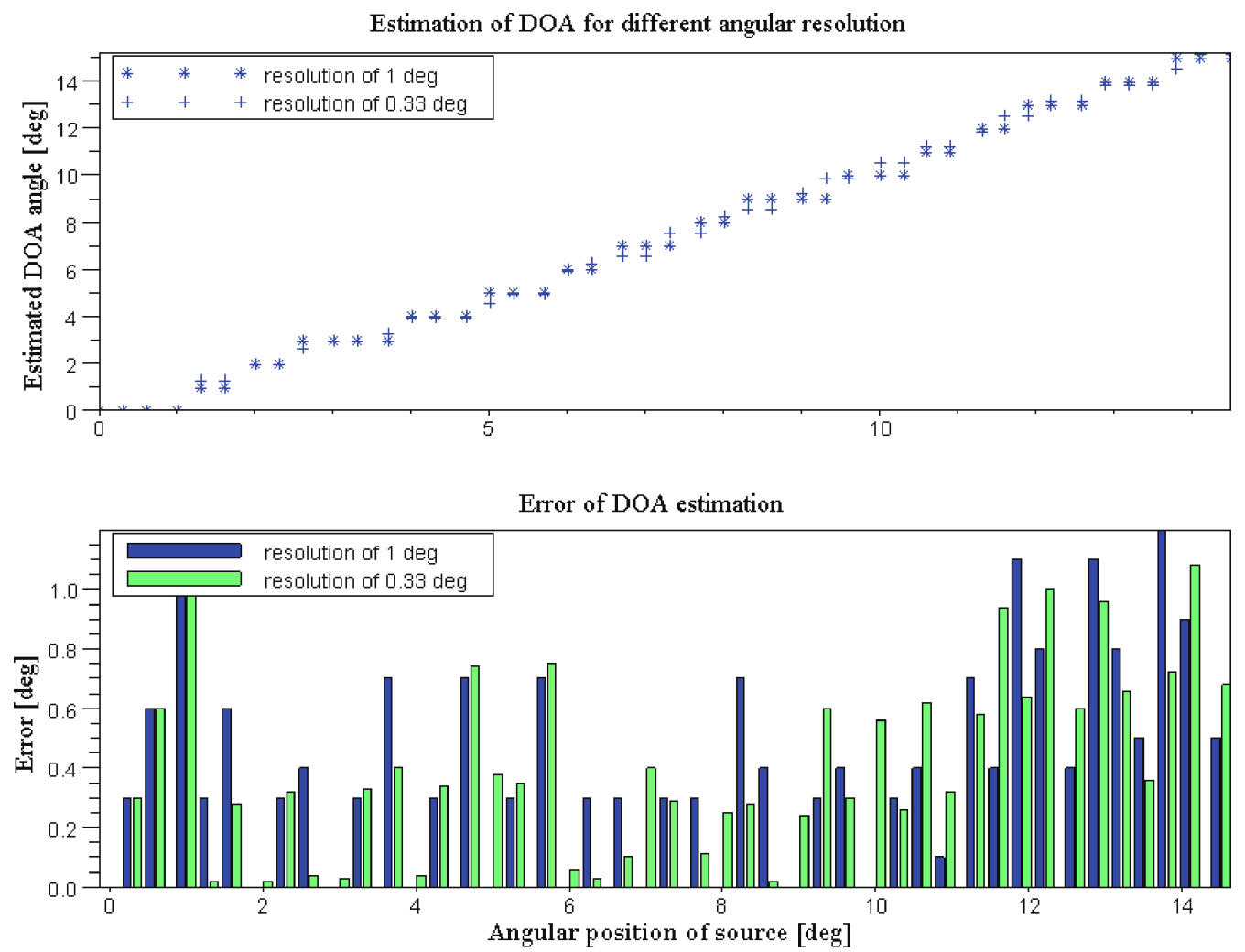

Fig. 9. Estimation results of direction of arrival (DOA) of ultrasound wave incidence for various source positions and various values of beam steering step, when delay-and-sum beamforming and correlation algorithm were used. Bar charts show estimation error.
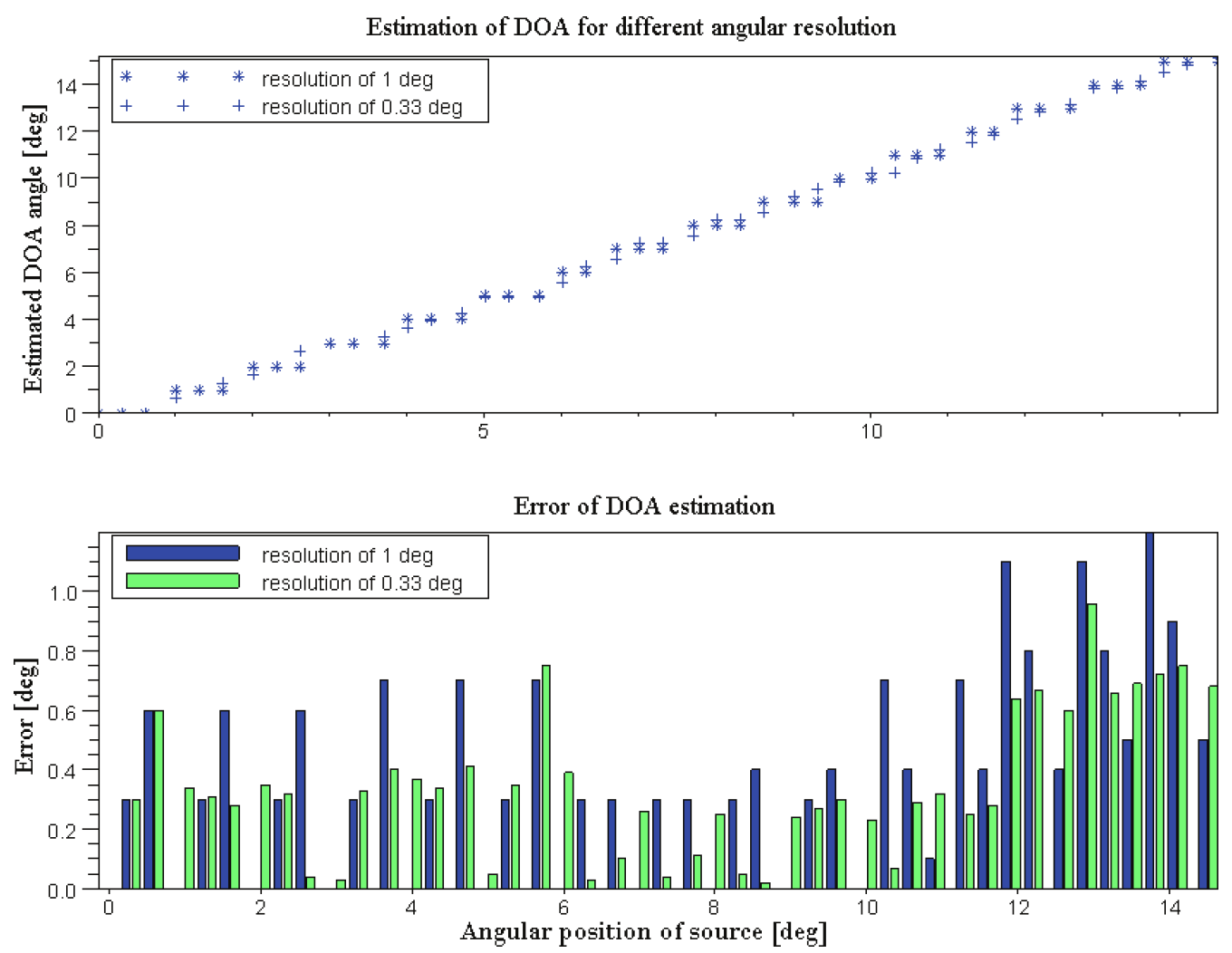

Fig. 10. Estimation results of direction of arrival (DOA) of ultrasound wave incidence for various source positions and various values of beam steering step, when interpolated delay-and-sum beamforming and correlation algorithm were used. Bar charts show estimation error. 

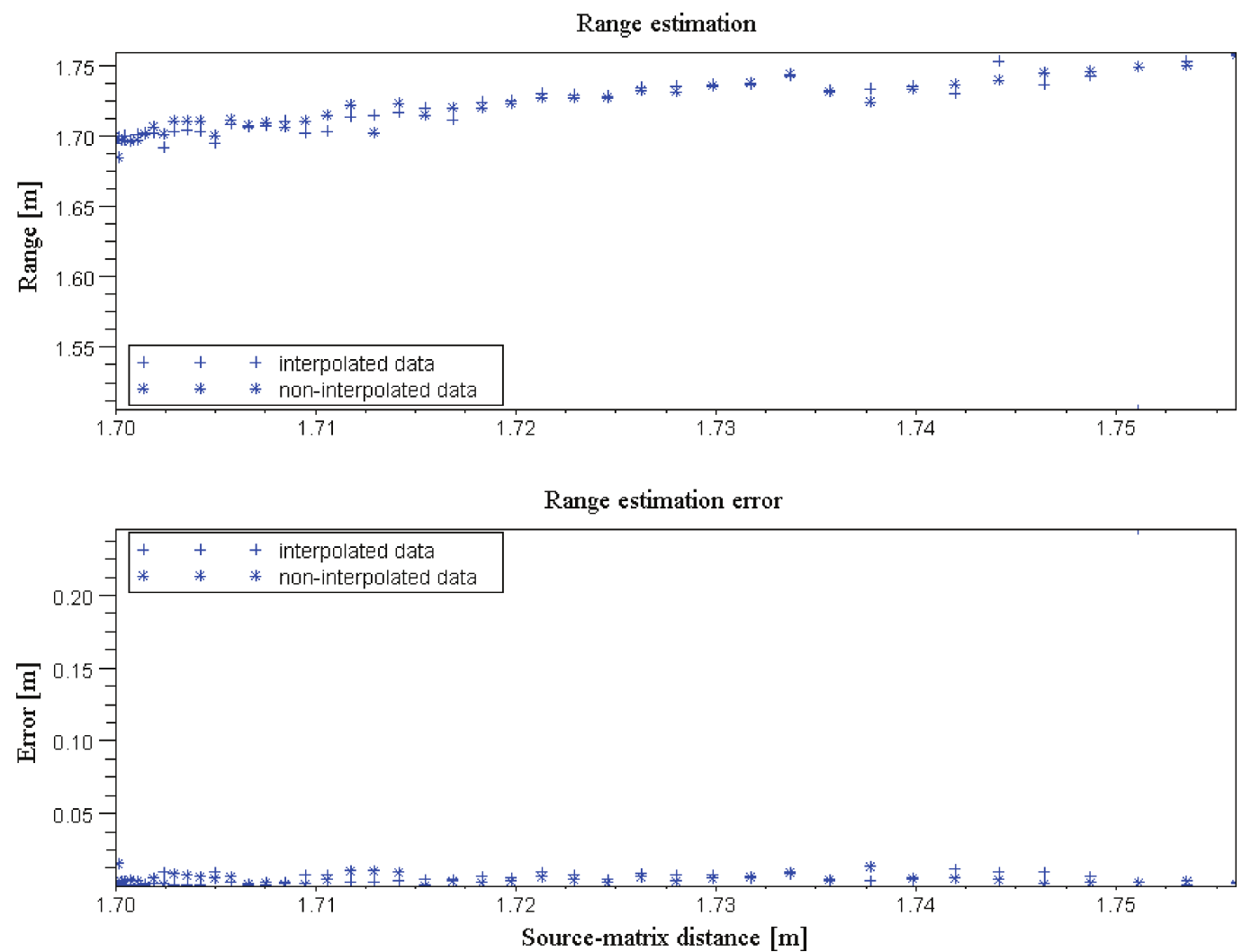

Fig. 11. Results of estimation of the source-matrix distance for various positions of the source, when no interpolated $($ marked $*)$ and interpolated $($ marked + ) delay-and-sum beamforming was used.
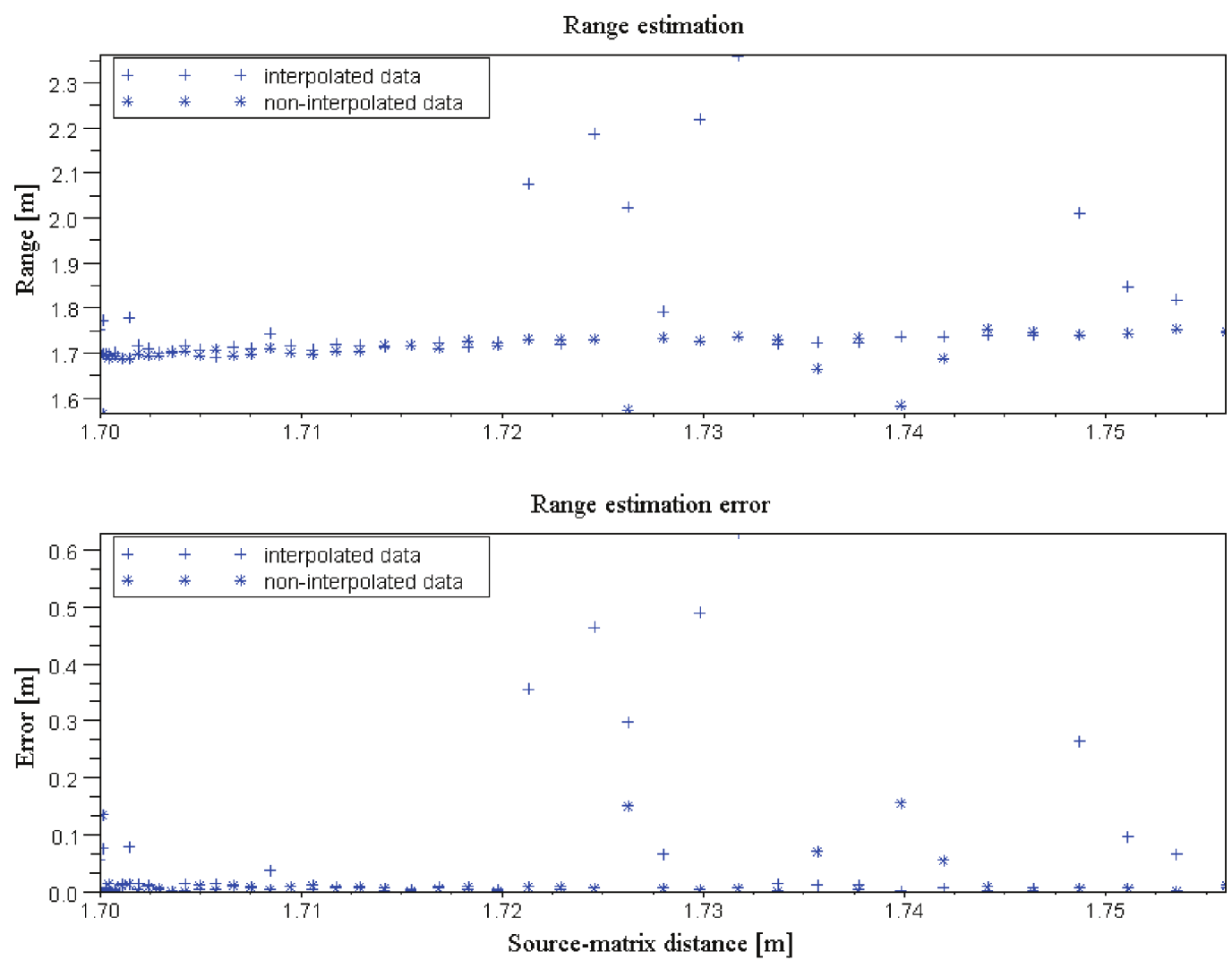

Fig. 12. Results of estimation of the source-matrix distance for various positions of the source, when no interpolated $\left(\right.$ marked $\left.{ }^{*}\right)$ and interpolated $($ marked + ) delay-and-sum beamforming and correlation was used. 
Change of angular position of the signal source results in alteration of source-array distance in accordance with Eq. (12):

$$
\begin{aligned}
d(p) & =\sqrt{r^{2}+\left(\frac{p}{100}\right)^{2}}, \\
p & =0 \ldots 45 .
\end{aligned}
$$

Results of estimation of this distance in the theoretical function of source-matrix distance and estimation error are shown in Fig. 11 and Fig. 12. The difference between the figures is a result of using different processing algorithms. For estimation presented in Fig. 11 the team used traditional delay-and-sum beamforming, and for results visible in Fig. 12 beamforming with function of correlation of the transmitted and received signal was used.

\subsection{Results for frequency modulated signals}

Unlike in case of the results presented in the previous sub-chapter, the results shown below were obtained using variable frequency signal. The results of estimation of ultrasonic wave incidence angle and the source-matrix distance were obtained using algorithms analogous to constant frequency signal.
The results obtained using delay-and-sum beamforming algorithm are shown in Fig. 13. As in case of Fig. 7, presented are results of calculations performed for two different values of angle $\Delta \varphi_{k}$ the successive beams were steered by.

As before Fig. 14 shows results of estimation of ultrasonic wave incidence angle. The difference is that this time the results were obtained using sample interpolation algorithm in accordance with Eq. (9). As in the case of beamforming with no interpolation, the team performed calculations for two different angle values $\Delta \varphi_{k}$. Figure 15 shows the results of estimation of ultrasonic wave incidence angle calculated using correlation of the transmitted and received signal and applying beamforming to the obtained time series. Similarly Fig. 16 shows the same result set, the only difference being that, these were obtained using upsampling and interpolation in addition.

Results of estimation of this distance in the theoretical function of source-matrix distance and estimation error are shown in Fig. 17 and Fig. 18. The difference between the figures is a result of using different processing algorithms. For estimation presented in Fig. 17 the team used traditional delay-and-sum beamforming, and for results visible in Fig. 18 beamforming with function of correlation of the transmitted and received signal was used.
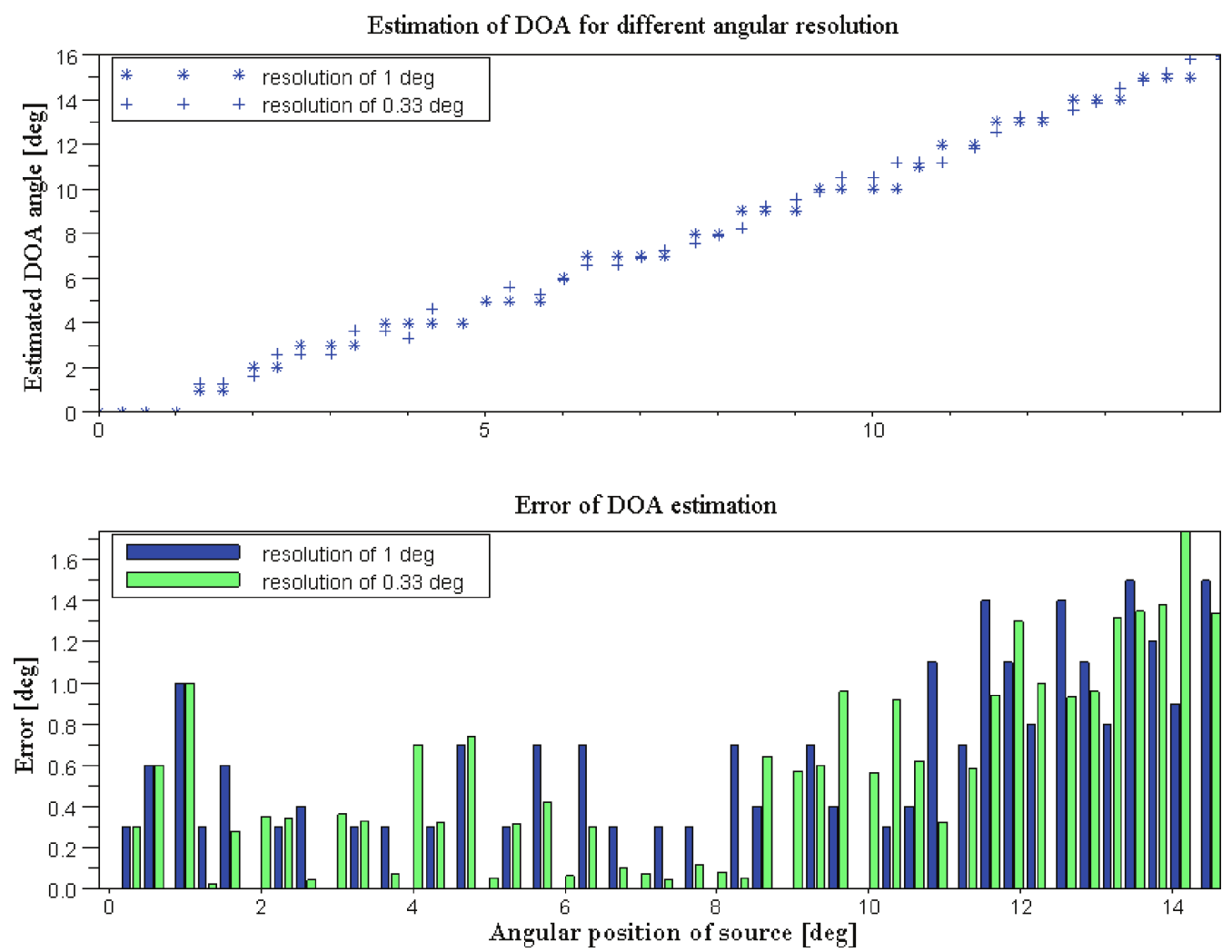

Fig. 13. Estimation results of direction of arrival (DOA) of ultrasound wave for various source positions and various values of beam steering step. Bar charts show estimation error. 

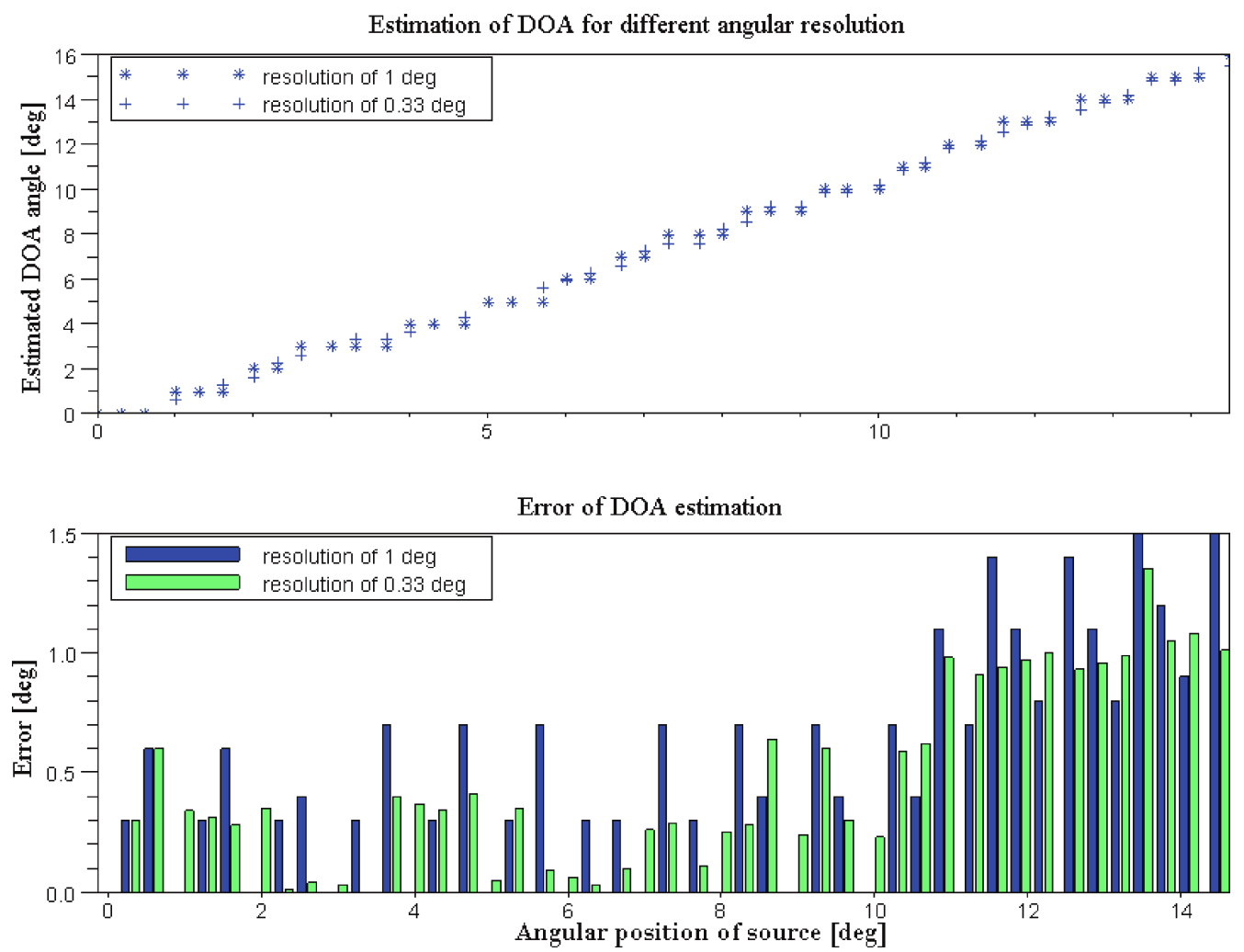

Fig. 14. Estimation results of direction of arrival (DOA) of ultrasound wave for various source positions and various values of beam steering step, when interpolated beamforming was used. Bar charts show estimation error.
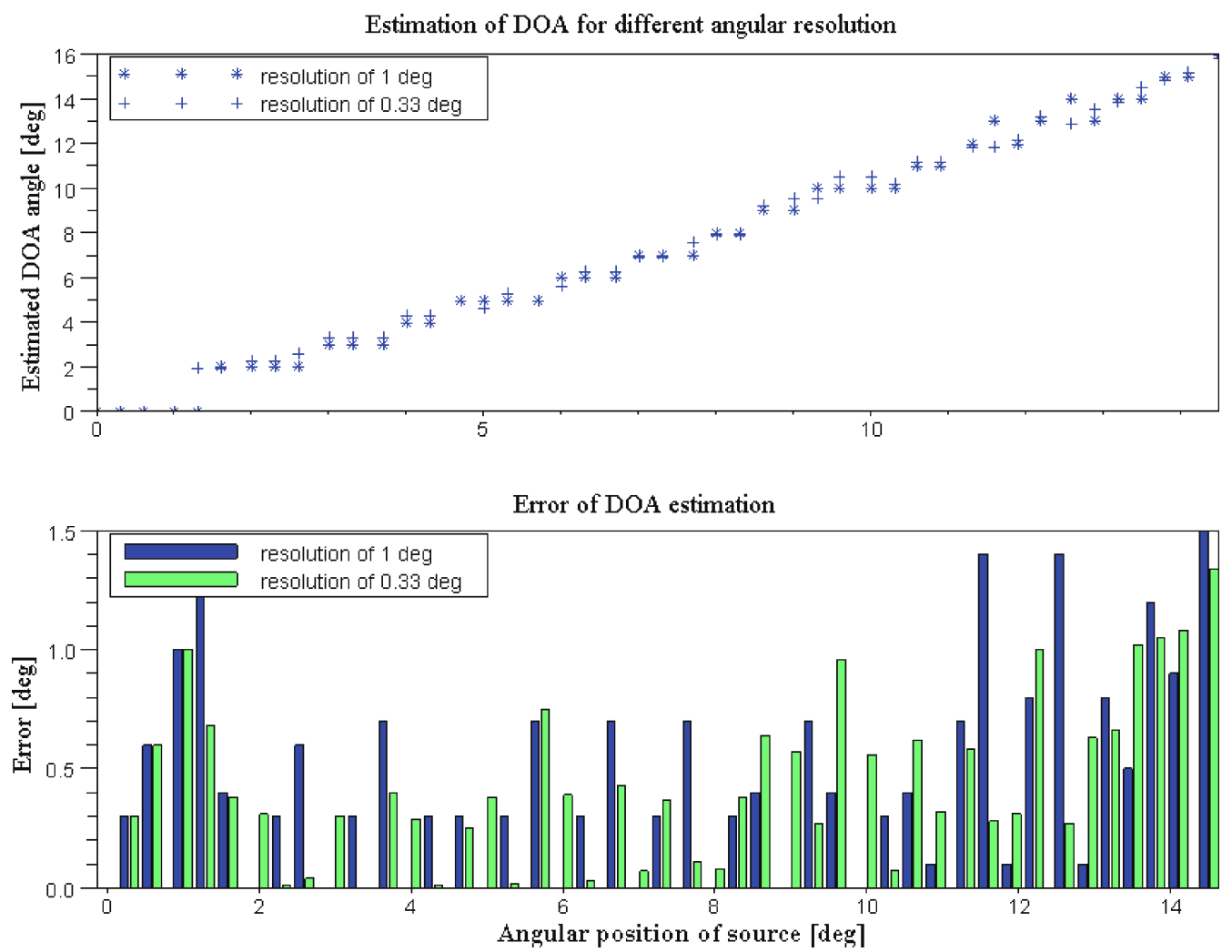

Fig. 15. Estimation results of direction of arrival (DOA) of ultrasound wave incidence for various source positions and various values of beam steering step, when delay-and-sum beamforming and correlation algorithm were used. Bar charts show estimation error. 
Estimation of DOA for different angular resolution
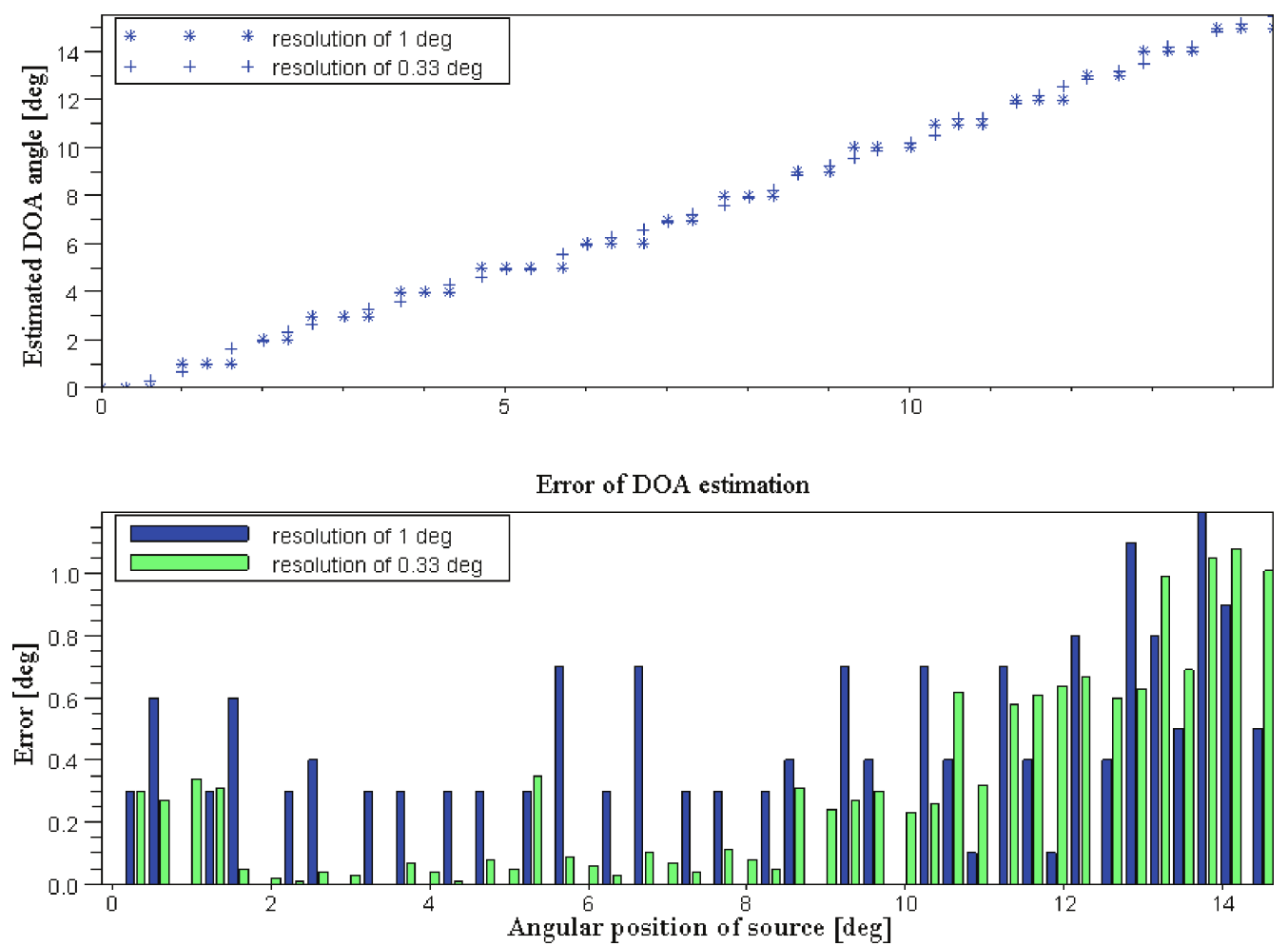

Fig. 16. Estimation results of direction of arrival (DOA) of ultrasound wave incidence for various source positions and various values of beam steering step, when interpolated delay-and-sum beamforming and correlation algorithm were used. Bar charts show estimation error.
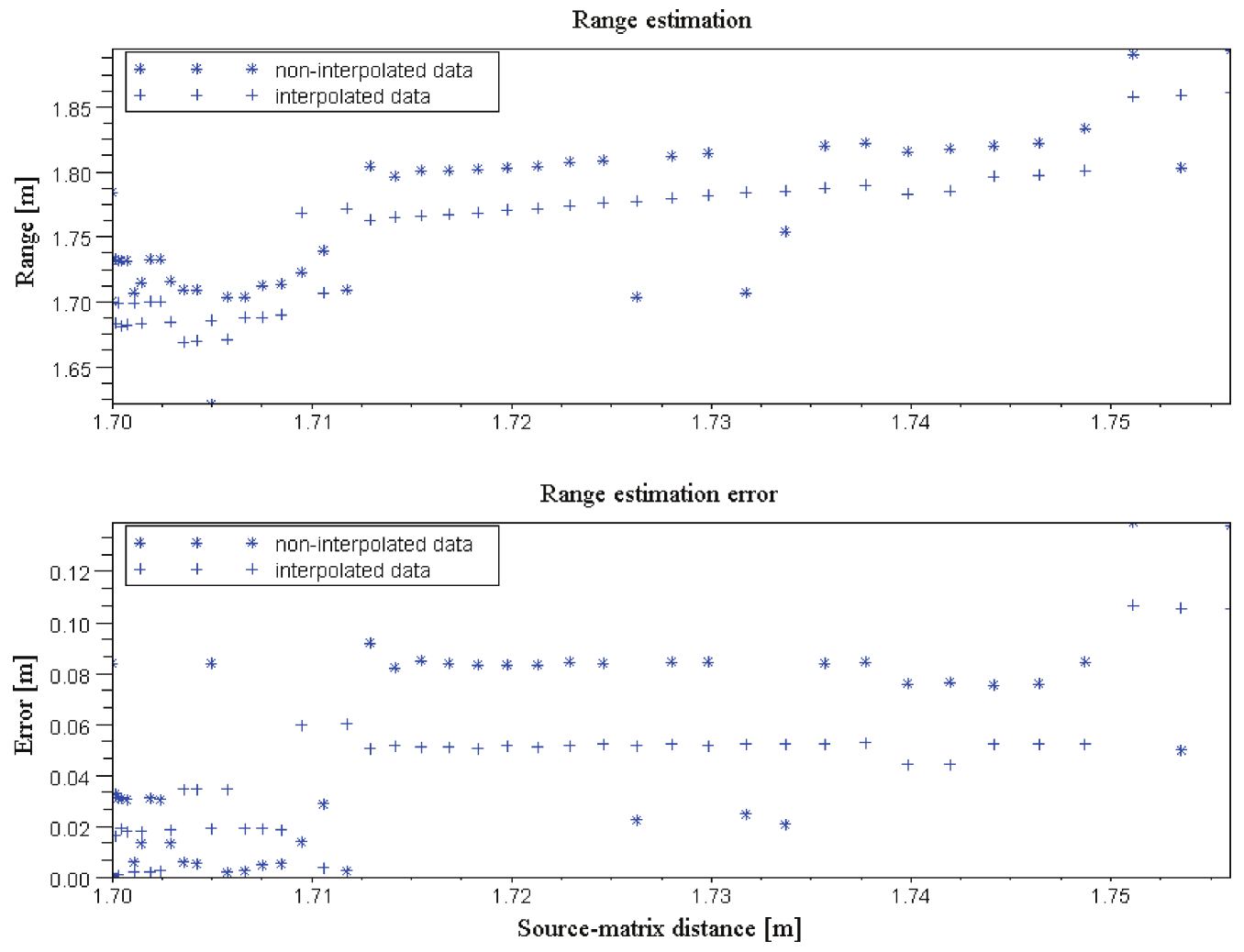

Fig. 17. Results of estimation of the source-matrix distance for various positions of the source, when no interpolated $(\operatorname{marked} *)$ and interpolated $($ marked + ) delay-and-sum beamforming was used. 


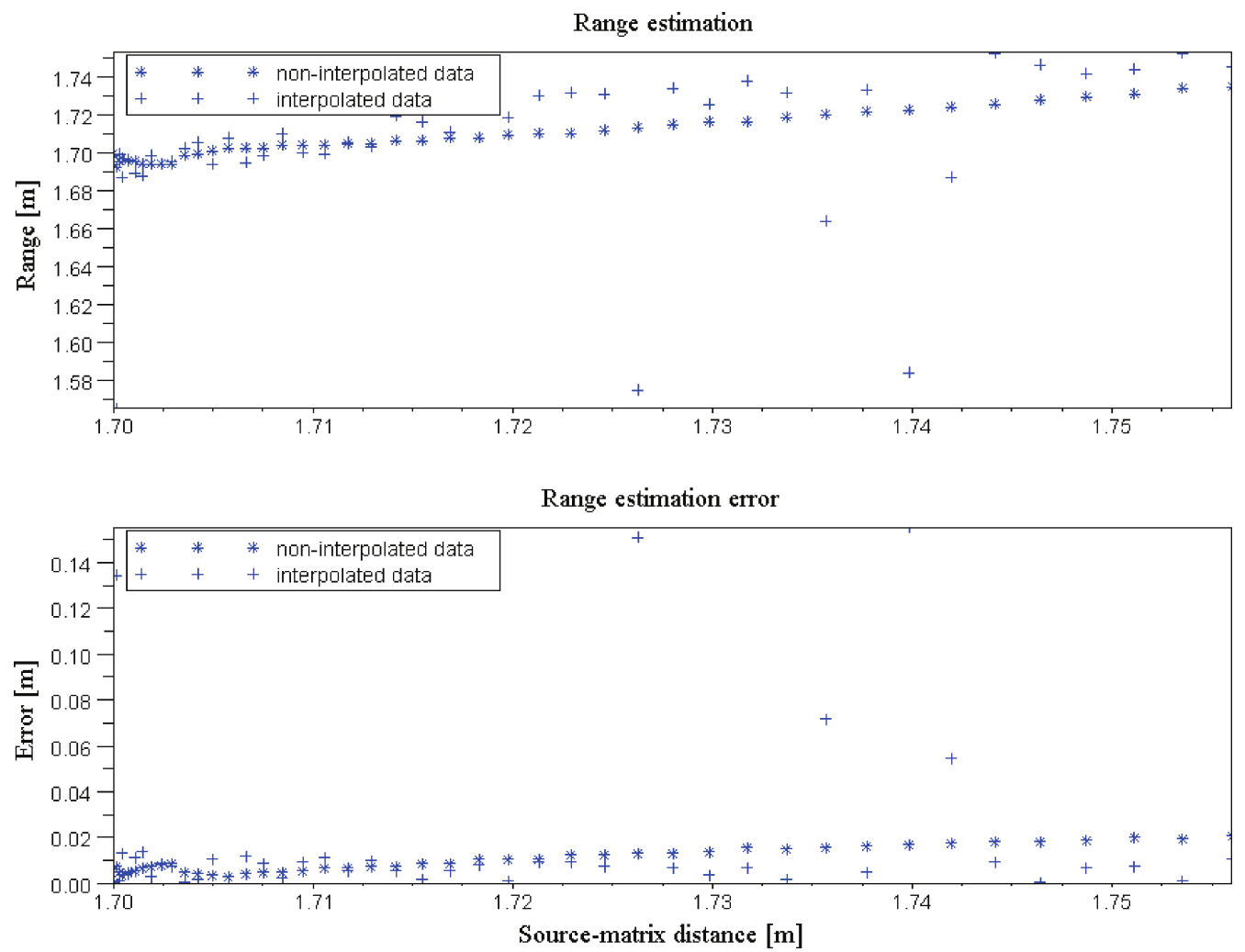

Fig. 18. Results of estimation of the source-matrix distance for various positions of the source, when no interpolated $\left(\right.$ marked $\left.{ }^{*}\right)$ and interpolated $($ marked + ) delay-and-sum beamforming and correlation was used.

Table 1. Mean squared error of source-matrix distance estimation for different signals and calculation methods.

\begin{tabular}{|c|c|c|c|c|}
\hline \multirow{2}{*}{} & \multicolumn{2}{|c|}{ Delay-and-Sum Beamforming } & \multicolumn{2}{c|}{ Correlation Beamforming } \\
\cline { 2 - 5 } & no interpolation & interpolation & no interpolation & interpolation \\
\hline CF & 0.02 & 0.04 & 0.16 & 0.04 \\
\hline FM & 0.06 & 0.05 & 0.01 & 0.04 \\
\hline
\end{tabular}

Table 2. Mean squared error of horizontal angle for different signals and calculation methods.

\begin{tabular}{|c|c|c|c|c|c|c|c|c|}
\hline \multirow{2}{*}{} & \multicolumn{3}{|c|}{ Delay-and-Sum Beamforming } & \multicolumn{3}{c|}{ Correlation Beamforming } \\
\cline { 2 - 9 } & \multicolumn{2}{|c|}{ no interpolation } & \multicolumn{2}{|c|}{ interpolation } & \multicolumn{2}{c|}{ no interpolation } & \multicolumn{2}{c|}{ interpolation } \\
\cline { 2 - 9 } & 0.33 & 1 & 0.33 & 1 & 0.33 & 1 & 0.33 & 1 \\
\hline CF & 0.43 & 0.53 & 0.38 & 0.43 & 0.51 & 0.53 & 0.42 & 0.51 \\
\hline FM & 0.7 & 0.72 & 0.60 & 0.70 & 0.56 & 0.63 & 0.44 & 0.48 \\
\hline
\end{tabular}

\subsection{Summary of measurements results}

Table 1 and Table 2 shows results presented as graphs in previous sub-chapters. The figures presented in the tables below are characterised by mean squared error for every type of measurements and can be a basis for conclusions about quantitative comparison of individual methods of estimation of both the ultrasonic wave incidence angle and the source-matrix distance.

\section{Discussion}

The performed analyses are related to estimation of the location of a source of a known ultrasonic signal in two-dimensional plane (range, horizontal angle). It should be noted that the experiments were performed for sound wave incidence angles in the range of 014.5 [deg] with a step of 1 [deg] or 0.33 [deg]. On the other hand, the width of the main lobe modelled using 
parameter PW is equal to 4.5 [deg] with no beam steering. An analysis of the possibilities of estimation of ultrasonic wave incidence angle shows that estimation uncertainty is in most cases below $1[\mathrm{deg}]$, increasing the number of beams by condensing parameter $\Delta \varphi_{k}$ results in lower mean squared error and more linear processing characteristic. It is also evident that upsampling results in decreased estimation error, both in case of traditional beamforming and beamforming with correlation of the transmitted and received signal. In case of estimation of the source-matrix distance, sample interpolation does not result in decreased position uncertainty for both CF and FM signal. After an analysis of Fig. 12, Fig. 18 and error results in Table 1, one can conclude that the results of distance estimation are best when modulated signal correlated with received signal is used. The values shown in Fig. 18, all of which are below $1.7[\mathrm{~m}]$, are a result of using setup geometry in which the centre of the setup for the minimum source-matrix distance d overlaps the geometric centre of the matrix. Specifically, the minimum distance $\mathrm{d}$ occurs, when the source is positioned at the point equal to $p=50 \times M \times D$.

\section{Conclusions}

The study involved analysis of the operation of a linear matrix and experimental research, the aim of which was to estimate the position of ultrasonic wave source in two-dimensional space, where angular position of the source in horizontal plane and matrixsource distance are the measures (range, horizontal angle). It can be concluded on the basis of the analysis of the obtained results of experimental research that the error of estimation of both angular position and source-matrix distance depends on the source signal parameters and processing algorithm of the signal recorded by the source. Additionally, it seems that the array is characterised by very good angular resolution and can discriminate the position of the signal source with accuracy of up to 1 [deg]. It is true that in case of source-matrix distance estimation, the best results were achieved, when using frequency modulated signals and correlation based signal processing methods. It is understandable if you take into consideration the characteristics of pulse-compression technology used in radars. In case of sonars used in hydrolocation and espacially location system operating in air environment, it is difficult to generate and receive frequency modulated signals. The use of a array of miniature broadband MEMS sensors, makes it possible to detect signals modelled on bats' signals, which are modulated in frequency domain. The result was better precision of estimation of distance with no change to uncertainty of estimation of the source angular position. It should also be concluded that, as far as digital processing of signals, upsampling and sample interpolation are of significant use at increased angular resolution of microphone antenna. However, when the aim is to minimise the error of estimation of both source angular position and matrix-source distance, it is more reasonable to use methods utilising correlation function combined with delay-and-sum beamforming.

The results presented in the work can be suitable, when designing devices that may be applied in space imaging with the use of echo ranging. This is because the next step of the research is to use the constructed array as a part of location system for objects in air environment.

\section{References}

1. Bass H.E., Sutherland L.C., Zuckerwar A.J., Blackstock D.T., Hester D.M. (1995), Atmospheric absorption of sound: Further developments, The Journal of the Acoustical Society of America, 97, $1,680-683$.

2. Borenstein J., Koren Y. (1988), Obstacle avoidance with ultrasonic sensors, IEEE Trans. on Robotics and Automation, 4, 2, 213-218.

3. Chou T.N., Wykes C. (1997), An integrated vision/ultrasonic sensor for $3 D$ target recognition and measurement, Proc. Sixth International Conference on Image Processing and Its Applications, 189-193, Dublin.

4. Johnson D.H., Dudgeon D.E. (1993), Array Signal Processing, Prentice-Hall, Englewood Cliffs.

5. Ealo J., Seco F., Jimenez A. (2008), Broadband EMFi-based transducers for ultrasonic air applications, IEEE Transactions on Ultrasonics Ferroelectrics and Frequency Control, 55, 4, 919-929.

6. Evans L.B., Bass H.E., Sutherland L.C. (1972), Atmospheric Absorption of Sound: Theoretical Predictions, The Journal of the Acoustical Society of America, 51, 5B, 1565-1575.

7. Gudra T., Furmankiewicz J., Herman K. (2011), Bats Sonar Calls and its Application in Sonar Systems [in:] Sonar Systems, Kolev N.Z. [Ed.], InTech - Open Access Publisher, pp. 209-234.

8. Harput S., Bozkurt A. (2008), Ultrasonic Phased Array Device for Acoustic Imaging in Air, IEEE Sensors Journal, 8, 11-12, 1755-1762.

9. KAY L. (1964), An ultrasonic sensing probe as a mobility aid for the blind, Ultrasonics, 2, 2, 53-59.

10. Krim H., Viberg M. (1996), Two decades of array signal processing research: the parametric approach, IEEE Signal Processing Magazine, 13, 4, 67-94.

11. Medina L., Wykes C. (2001), Multiple target 3D location airborne ultrasonic system, Ultrasonics, 39, 1, 19-25.

12. Paajanen M., Lekkala J., Kirjavainen K. (2000), ElectroMechanical Film (EMFi) - a new multipurpose electret material, Sensors and Actuators A: Physical, 84, 1-2, 95-102. 
13. Peremans H., Audenaert K., Van Campenhout J.M. (1993), A high-resolution sensor based on tri-aural perception, IEEE Trans. on Robotics and Automation, $\mathbf{9}$, $1,36-48$.

14. Salamon R., Kowalik R., Podstawka I. (1997), Detection and signal processing in the acoustic echolocation system for the blind, Archives of Acoustics, 22, 277-295.

15. Schillebeeckx F., De Mey F., Peremans H. (2008), Bio-inspired sonar antennae: Enhancing directivity patterns for localization, Proc. 2nd IEEE RAS \& EMBS International Conference on Biomedical Robotics and Biomechatronics, pp. 366-371, USA.

16. Shi R.Z., Horiuchi T.K. (2007), A Neuromorphic VLSI Model of Bat Interaural Level Difference Process- ing for Azimuthal Echolocation, IEEE Transactions on Circuits and Systems, 54, 1, 74-88.

17. Strąkowski M.R., Kosmowski B.B., Kowalik R., WierzBa P. (2006), An ultrasonic obstacle detector based on phase beamforming principles, IEEE Sensors Journal, 6, 1, 179-186.

18. Van Veen B.D., Buckley K.M. (1988), Beamforming: a versatile approach to spatial filtering, IEEE Acoustics, Speech, and Signal Processing, 5, 2, 4-24.

19. WebB P., Wykes C. (1996), High-resolution beam forming for ultrasonic arrays, IEEE Transactions on Robotics and Automation, 12, 1, 138-146.

20. Wykes C., Nagi F., WebB P. (1993), Ultrasound imaging in air, Proc. International Conference on Acoustic Sensing and Imaging, pp. 77-81, London. 